\title{
Chromosome in situ suppression hybridisation in clinical cytogenetics
}

\author{
M A Hulten, C P Gould, A S H Goldman, J J Waters
}

\begin{abstract}
The use of chromosome in situ suppression hybridisation with whole chromosome libraries has previously been reported by various research laboratories to be an effective method of identifying specific human chromosomal material. As a clinical cytogenetic service laboratory we have used the technique as a complement to diagnosis by classical chromosome banding. In three examples of structural rearrangements the potential use of the "chromosome painting' method is assessed for its ability to enhance the routine cytogenetic service currently available.
\end{abstract}

A number of recent reports have shown that nonisotopic chromosome in situ suppression (CISS) hybridisation using flow sorted chromosome libraries, so called 'chromosome painting', may become an important diagnostic tool in clinical cytogenetics. ${ }^{1-6}$ The majority of these reports, however, emanate from research laboratories, and the adaptation to their application as a routine procedure in the Service laboratory has not been documented. In this paper we illustrate with three clinical cases our experience with introducing fluorescence in situ hybridisation as an adjunct to traditional banding techniques in the diagnosis of constitutional chromosome abnormalities.

\section{Materials and methods \\ CELL PREPARATIONS}

Metaphase spreads of human chromosomes were prepared by standard techniques. ${ }^{7}$ Fixed suspen-

West Midlands Regional Genetics Service, East Birmingham Hospital, Bordesley Green East, Birmingham B9 5ST.

M A Hulten, C P Gould, A S H Goldman, J J Waters

Correspondence to Professor Hulten.

Received for publication 11 April 1991

Accepted for publication 15 May 1991. sions, in methanol:acetic acid $(3: 1)$, were fresh or had been stored at $-20^{\circ} \mathrm{C}$ for up to 18 months. Slides that had been precleaned by the manufacturer (Menzelglaser, Germany) were used without further pretreatment. Slides made for hybridisation on the same day were baked at $65^{\circ} \mathrm{C}$ for one to two hours before use. Slides that were to be processed within one to six weeks were stored at $-20^{\circ} \mathrm{C}$.

Pre- $G$ banding, when required, was carried out by the Trypsin-Leishman (GTL) method. Metaphases were photographed and positions recorded. Slides were destained in methanol:acetic acid (3:1), washed in PBS for 10 minutes, fixed in $2 \%$ paraformaldehyde in $50 \% 0.1 \mathrm{~mol} / 1 \mathrm{MgCl}_{2}$ and $50 \%$ PBS for 10 minutes, then dehydrated by an alcohol series $(50 \%, 70 \%, 100 \%$ ethanol), and taken through the hybridisation procedure.

DNA LIBRARIES AND LABELLING

Whole chromosome libraries, cloned in bluescribe plasmids, were a gift from Dr J W Gray of the Lawrence Livermore Laboratories, California. ${ }^{1}$ Plasmids were isolated from host cells using calcium chloride gradients. Qiagen columns (DIAGEN, Dusseldorf, Germany) were used for isolation of pBS-3. Labelling of whole plasmid was carried out as per kit instructions (BRL) using biotin-11-dUTP (Sigma) as the labelled nucleotide. Sephadex G50 column separation of unincorporated nucleotides was not applied.

CISS HYBRIDISATION AND PROBE DETECTION

The CISS hybridisation method in our laboratory is based on the methods described by Pinkel et al $^{4}$ as 'protocol 1'. Hybridisation conditions were investigated for each library and the method adapted accordingly. Chromosome specific probes were prehybridised at $37^{\circ} \mathrm{C}$ with competitor DNA (human placental DNA sonicated to $500 \mathrm{bp}$ fragments) before the mixture was hybridised in 50\% formamide, $10 \%$ dextran sulphate, and $2 \times$ SSC to metaphase spreads on slides at $37^{\circ} \mathrm{C}$. In each case four parameters were considered: (1) amount of chromosome probe per hybridisation spot, (2) probe DNA/ 
competitor DNA ratio, (3) prehybridisation time (if any), and (4) hybridisation time. Optimised conditions for each probe are outlined in the table. In our experience these conditions are variable and they are presented only as a guide.

Microscopy was carried out by a Zeiss Axioplan microscope using the fluorescence filters 09 and 15 . Photography was performed with Fujichrome 400 ASA colour slide film uprated to 800 ASA.

\section{Results}

CASE 1

The first case illustrates the potential of chromosome painting for resolving ambiguities in breakpoint identification in structural chromosome rearrangements. This case of an apparently simple balanced reciprocal translocation involving chromosomes 6 and 15 (fig 1A) is of particular interest, as the subject is suffering from Marfan syndrome (Child, personal communication). Chromosomes were pre-G banded (fig 2A) and then 'painted' with the chromosome 15 library (fig 2B). Fig 2C shows the counter hybridisation with the number 6 library. The breakpoints were tentatively assigned with $G$ banding as either $\mathrm{t}(6 ; 15)(\mathrm{q} 13 ; \mathrm{q} 24)$ or $\mathrm{t}(6 ; 15)(\mathrm{q} 15 ; \mathrm{q} 26.2)$. Chromosome painting showed that the latter interpretation is correct. The relevance of these breakpoints to Marfan syndrome remains to be determined. ${ }^{8}$

CASE 2

The second case illustrates the delineation of a much rarer constitutional structural rearrangement, an insertional translocation involving three breakpoints. The ascertainment of this case was by the routine screening of a child with developmental delay and dysmorphism. $G$ banding identified extra material inserted into the long arm of chromosome 7 (fig 1B). The karyotype was tentatively assigned as 46,XY, - 7, + dir ins(7;6)(q21.2;?q16.2q23.1). Chromosome painting with the 6 library (fig $3 \mathrm{~A}$ ) and the 7 library (fig 3B) confirmed the identity and the location of the insertion of $6 \mathrm{q}$ material into $7 \mathrm{q}$. The father was found to be a balanced carrier of the insertion.

CASE 3

This case illustrates the use of chromosome painting to identify extra material of unknown origin. An institutionalised adult male with mental retardation and slight facial dysmorphism was found to have extra material present on the short arm of chromosome 3 (fig 1C). Chromosome painting with the 3 library (fig 3C) and the 8 library (fig 3D) showed that the extra material in the terminal region of the $3 p$ arm is derived from chromosome 8 . On the basis of the $\mathrm{G}$ banding pattern and chromosome painting we were then able to interpret the karyotype as $46, \mathrm{XY},-3,+\operatorname{der}(3) \mathrm{t}(3 ; 8)(\mathrm{p} 26 ; \mathrm{p} 22)$. Blood from the parents was unavailable. Other probes, which were possible candidates for the origin of the extra material, were also applied and excluded: pBS-4, 6, 7, 13 (results not shown).

\section{Discussion}

We have found the chromosome specific libraries straightforward to use for in situ hybridisation, providing a diagnostic adjunct to classical metaphase analysis. In this report we have shown the potential of the 'chromosome painting' technology for identifying the breakpoints in structural rearrangements, such as reciprocal and insertional translocations, where classical high resolution $G$ banding may remain ambiguous. We have also found chromosome painting helpful for the identification of the origin of chromosome segments, which have remained unidentified by $G$ banding, but this may be labour intensive, as a systematic approach by in situ hybridisation with a number of chromosome specific probes is then required.

One advantage in using the chromosome specific probes for in situ hybridisation is the high efficiency of labelling of individual metaphases. In our experience this approaches $100 \%$, which compares favourably with the much lower rate of labelling that may be achieved when using single copy probes. This makes the chromosome painting technique a valid approach to be exploited for the identification of chromosome mosaics. It is of special importance to note the high efficiency of metaphase labelling seen even when dealing with relatively small chromosome segments, such as the $15 \mathrm{q} 26.2-$ ter in case 1 and the terminal $\mathrm{Yp}$ segment in an XX male (unpublished observations). It will be of interest to find out more about the relation between the size of the chromosome segment and the labelling efficiency to answer outstanding questions, such as the size of the smallest segment, that will still retain a high rate of labelling. This resolution will vary between chromosomes and chromosome segments, as successful hybridisation requires modification of both concentration of individual chromosome probes and competitor and variation in the timing of prehybridisation/hybridisation (table). The variation in the amount of competitor DNA required to suppress cross hybridisation may of course reflect underlying differences in sequence homology between individual chromosomes.

Figure 1 Partial karyotypes by $G$ banding. Arrows indicate breakpoints. (A) Case $1: t(6 ; 15)$. The $G$ banding indicates the breakpoints of chromosomes 6 and 15 at q13 or $q 15$, and $q 24$ or $q 26.2$, respectively. They were subsequently defined by chromosome painting at $q 13$ and q26.2 (fig 2). (B) Case 2: dir ins $(7 ; 6)(q 21.2 ; q 16.2 q 23.1)$. These breakpoints were tentatively identified by $G$ banding and confirmed by chromosome painting ( fig 3). (C) Case 3: $3 p+$. The $G$ banding identifies the abnormal chromosome 3. The origin of the extra $3 p$ material from chromosome 8 was determined by chromosome painting (fig 3 ). 
(A)

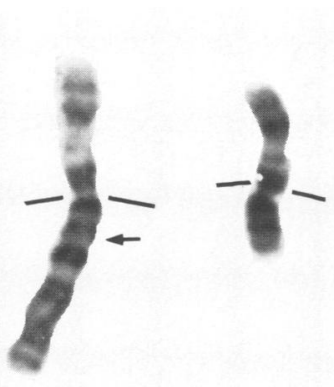

(B)
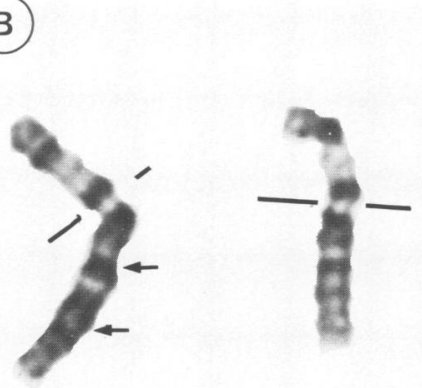

(C)

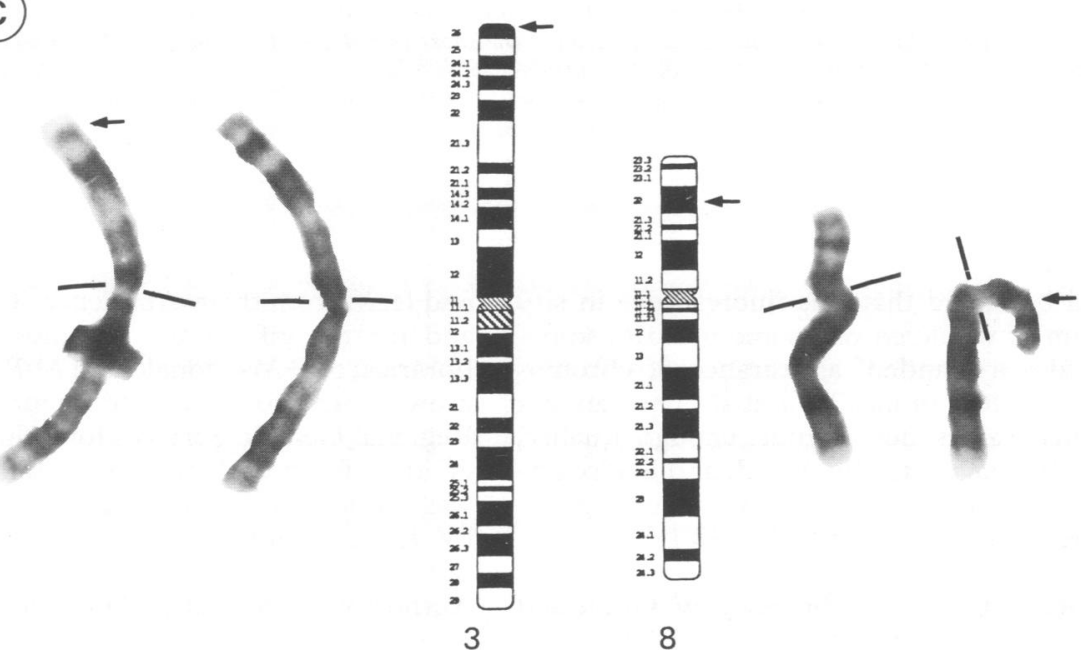

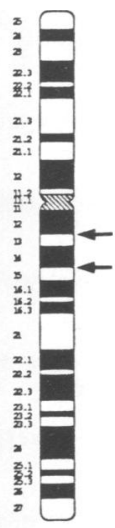

6
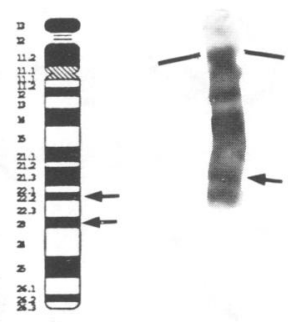

15

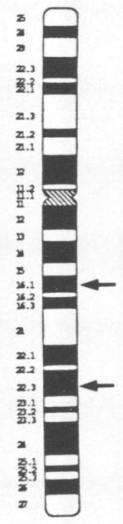

6
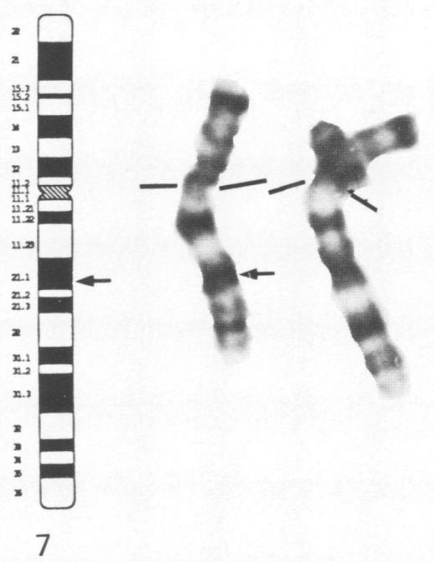

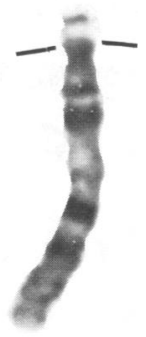



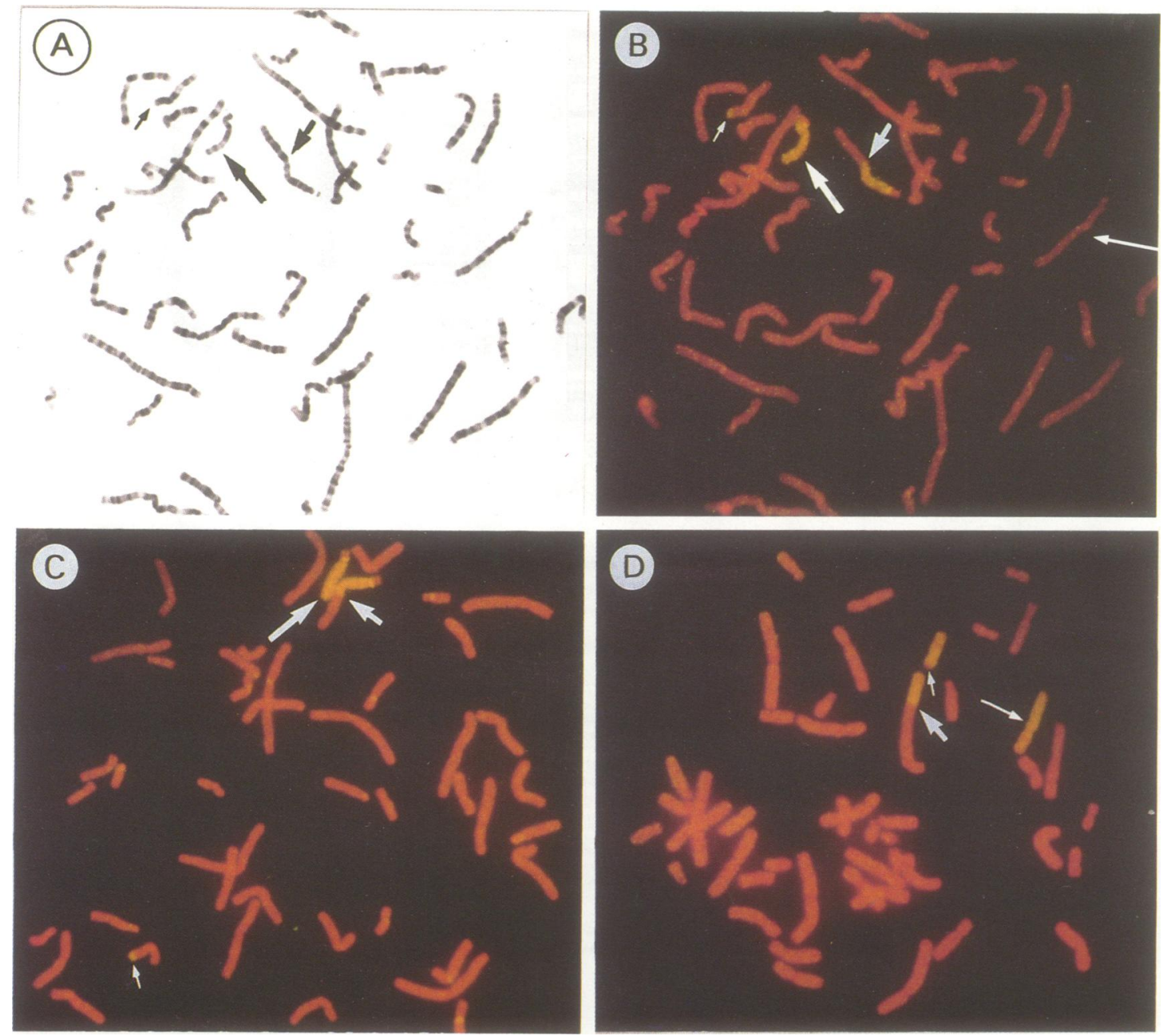

Figure 2 Case 1: 46,XY,t(6;15) (q15;q26.2). Consecutive stainings of the same metaphase spread by $G T L(A)$ and the chromosome 15 library (B). Two further metaphases, hybridised with the chromosome $15(C)$ and the chromosome 6 libraries (D). Note the short translocated chromosome 15 segment (short thin arrow), and the reciprocal translocation product with a large chromosome 6 segmeni (short bold arrow), the normal chromosome 6 (long thin arrow), and the normal chromosome 15 (long bold arrow).

It should be added that this fluorescence in situ hybridisation of whole chromosome libraries sometimes provides a 'banded' appearance of chromosomes, as illustrated in figs $3 \mathrm{~A}$ and $\mathrm{B}$. The banding obtained this way is not of high enough quality, however, to be diagnostically useful, and in circumstances where banding is essential it is more appropriate to preband chromosomes by GTL.

We are grateful to Drs D Pinkel, J W Gray, and R Segraves for allowing us to visit their laboratory and learn from their experience (CPG and ASHG), and for the gift of the chromosome specific pBS libraries; to F Macdonald and M Rindl for advice on growing up probes; and to members of staff of the Regional Genetic Services for help at various stages of identifying and processing samples. This work was supported by L F Saugstad's research fund and by travel grants from the Competitive Bursary Scheme of the West Midlands Regional Health Authority (CPG) and American Express Travel Agency (ASHG). 

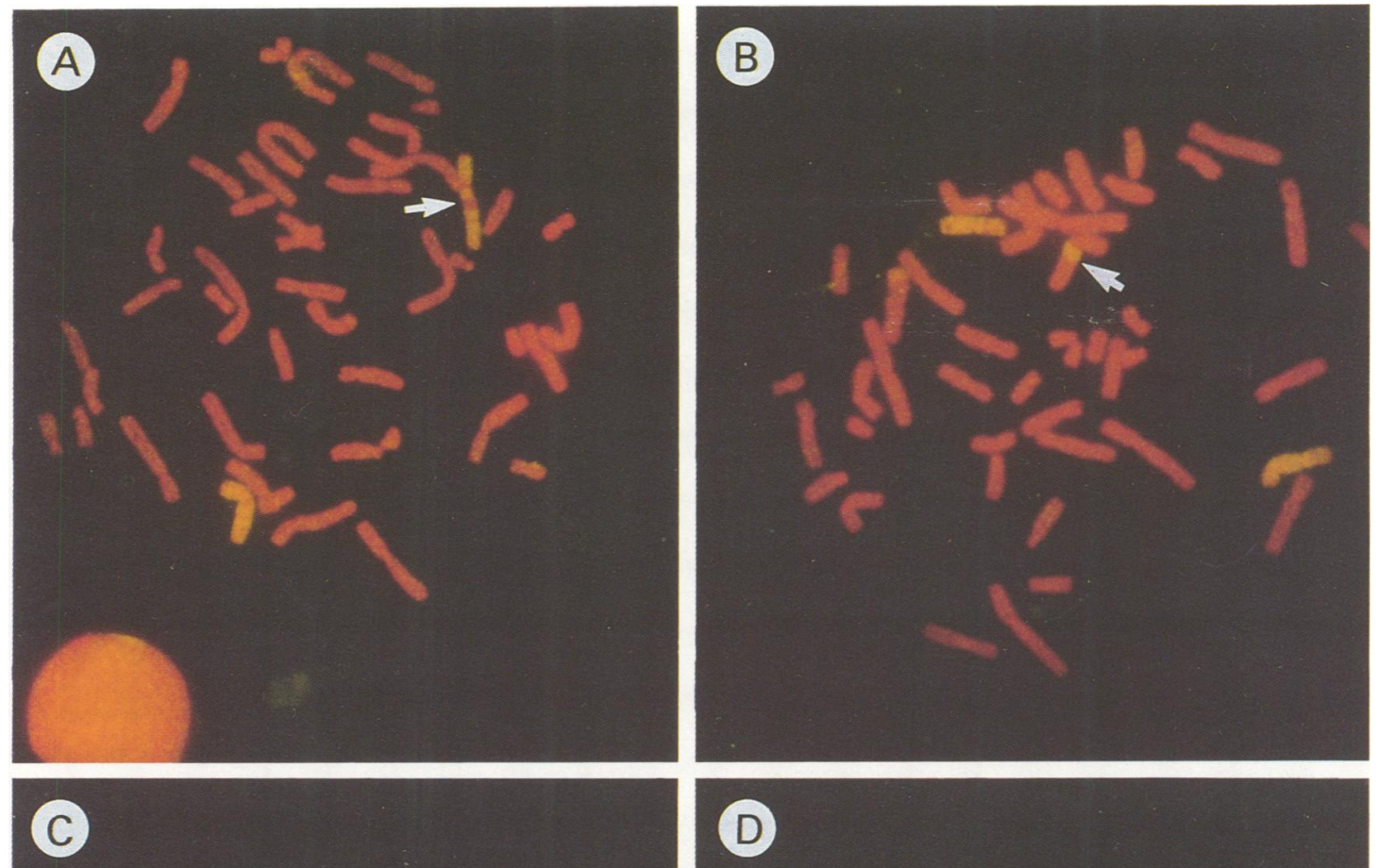
Typical DNA concentrations and hybridisation times used for specific chromosome libraries with minimal cross hybridisation.

\begin{tabular}{lcccc}
\hline Chromosome & $\begin{array}{c}\text { Probe } \\
(\mathbf{n g} / 10 \mu \mathrm{l})\end{array}$ & $\begin{array}{c}\text { Competitor } \\
(\mathbf{n g} / 10 \mu \mathrm{l})\end{array}$ & $\begin{array}{c}\text { Prehybridisation } \\
(\mathbf{m i n})\end{array}$ & $\begin{array}{c}\text { Hybridisation } \\
(\mathbf{h})\end{array}$ \\
\hline 1 & 60 & 100 & 0 & 16 \\
2,3 & 60 & 50 & 0 & 16 \\
4 & 30 & 500 & 30 & 16 \\
6 & 90 & 1000 & 40 & 16 \\
7 & 60 & 1000 & 0 & 64 \\
8 & 60 & 50 & 0 & 16 \\
9 & 60 & 100 & 30 & 16 \\
10 & 60 & 0 & 16 \\
11,12 & 60 & 50 & 40 & 16 \\
13,14 & 60 & 1000 & 0 & 16 \\
15 & 60 & 1500 & 0 & 64 \\
16,17 & 60 & 100 & 0 & 16 \\
18 & 60 & 50 & 60 & 16 \\
19,20 & 60 & 50 & 0 & 16 \\
21 & 60 & 1500 & 0 & 16 \\
$\mathbf{X}$ & 60 & 50 & 40 & \\
Y & 60 & 10 & 0 & \\
\hline
\end{tabular}

The chromosome 5 library is unavailable at present.

1 Cremer $T$, Lichter $P$, Borden J, Ward DC, Manuelidis I. Detection of chromosome aberrations in metaphase and interphase tumour cells by in-situ hybridization using chromosome-specific library probes. Hum Genet 1988;80:235-46.

2 Lichter P, Cremer T, Borden J, Manuelidis L, Ward DC. Delineation of individual human chromosomes in metaphase and interphase cells by in-situ suppression hybridization using recombinant DNA libraries. Hum Genet 1988;80:224-34.

3 Lichter P, Cremer T, Chang Tang CJ, Watkins PC, Manuelidis L, Ward DC. Rapid detection of chromosome 21 aberrations by in-situ hybridization. Proc Natl Acad Sci USA 1988; 85:9664-8.

4 Pinkel D, Lanlegent J, Collins C, et al. Fluorescence in situ hybridization with human chromosome-specific libraries: de- tection of trisomy 21 and translocations of chromosome 4. Proc Natl Acad Sci USA 1988;85:9138-42.

5 Arnoldus EPJ, Wiegant J, Noordermeer IA, et al. Detection of Philadelphia chromosome in interphase nuclei. Cytogenet Cell Genet 1990;54:108-11.

6 Jauch A, Oaumer C, Lichter P, et al. Chromosomal in-situ suppression hybridization of human gonosomes and autosomes and its use in clinical cytogenetics. Hum Genet 1990;85:145-50.

7 Rooney DE, Czepulkowski BH. Human cytogenetics: a practical approach. Oxford: IRL Press, 1986.

8 Dietz HC, Pyeritz RE, Hall BD, et al. The Marfan syndrome locus: confirmation of assignment to chromosome 15 and identification of tightly linked markers at $15 \mathrm{q} 15-\mathrm{q} 21.3$. Genomics 1991;9:355-61. 